\title{
Molecular Dynamic Simulation Study on Glass Transition Temperature of DGEBA-THPA/SWCNTs Composites
}

\author{
Cai Jiang, Jianwei Zhang, Shaofeng Lin, Dazhi Jiang \\ College of Aerospace Science and Engineering, National University of Defense Technology, Changsha, China \\ Email: jiangdz@nudt.edu.cn
}

Received October 2013

\begin{abstract}
Molecular dynamic (MD) simulations were carried out to predict the thermo-mechanical properties of the cured epoxy network composed of diglycidyl ether bisphenol A (DGEBA) epoxy resin and tetrahydrophthalic anhydride (THPA) curing agent and their single-walled carbon nanotubes (SWCNT) reinforced the epoxy matrix composites. Different characters such as the density of the materials and mean square displacements (MSDs) were calculated to estimate the glass transition temperatures (Tgs) of of the materials. $365 \mathrm{~K}$ and $423 \mathrm{~K}$ of the Tgs were obtained respectively, whereas the latter is much higher than the former. The simulation results indicated that the incorporation of SWCNTs in the epoxy matrix can significantly improve the Tg of the cured epoxy. The approach presented in this study is ready to be applied more widely to a large group of candidate polymers and nanofillers.
\end{abstract}

\section{KEYWORDS}

\section{Molecular Dynamics Simulation; Glass Transition Temperature; Carbon Nanotubes Composites}

\section{Introduction}

Glass transition temperature (Tg) is a key descriptor to evaluate the thermal properties of the heat-resistant materials, while the useability determined by the mechanical properties at high temperature. The ability to predict the $\mathrm{Tg}$ and mechanical properties is of great value in the selection and design of new materials. Conducting experiments to measure the $\mathrm{Tg}$ and mechanical properties is a reliable, however, time-consuming and expensive approach. Recently, molecular dynamics (MD) simulation has provided great insight into the Tg and elastic response of polymer and its composite materials.

Abu-Sharkh [1] conducted the rigid unit model and the explicit atom model to generate volume-temperature (V$\mathrm{T}$ ) data of poly (vinylchloride) respectively, which confirmed the validity of MD simulation in predicting the Tg of amorphous polar polymers. Wu et al. [2,3] calculated the density and elastic constants of diglycidyl ether bisphenol A (DGEBA) cured with isophorone diamine (IPD) using atomistic molecular simulation. The results indicated that both the use of COMPASS force-field and DREIDING force-field resulted in unrealistically high elastic constants whereas the former compared more favorably with the corresponding experimental values than the later. Fan et al. [4] used PCFF force-field to predict the Tg, linear thermal expansion coefficients (LCTEs) and Young's modulus of cross-linked EPON862-TETA (triethylenetetramine) systems from MD simulations. Their results were in good agreement with the experimental values in the literature. Li et al. [5,6] and Bandyopadhyay et al. [7,8] studied the EPON862-DETDA (diethylene toluene diamine) systems. The simulation results indicated a significant increase in Tg, Young's modulus and yield stress with degree of polymerization, while the thermal expansion coefficient (CTE) decreased with the overall crosslink density, and the yield strain was less sensitive to it, however, there was no discernible influence of cross-link distribution on the elastic modulus and the LCTE. Shenogina et al. [9,10] employed a new method-dynamic deformation approach to simulate the thermo-mechanical constants and the elastic constants of DGEBA-DETDA systems. Results were in very good agreement with experimental data of actual cured polymers. The approach showed excellent improvement compared to constants calculated using the static deformation approach.

Epoxy resin matrix composites are of special interest in the aerospace industry for the current andfuture aircraft and spacecraft due to their good heat-resistance and outstanding mechanicalproperties, comparing to other 
lightweight structural materials. It has been observed that the incorporation ofCNTs[11] in the epoxy resin can significantly enhance its mechanical, thermal, and electricalproperties, and thus, CNTs can be used as a potential reinforcement for epoxy. Gou et al. [12] investigated the interfacial bonding of single-walled carbon nanotube (SWCNT) reinforced epoxy resin composites in terms of stress transfer. A 250\% 300\% increase in storage modulus with the addition of $20 \sim 30 \mathrm{wt} \%$ nanotubes was resulted. Liang et al. [13] found that both the EPON862 resin and DETDA molecules have attractive intercalations with $(10,10)$ SWCNT, and the aromatic ring structures of the molecules try to align the aromatic ring planes toward the SWCNT surface and wrap around it. Mittal et al. [14] found that the reinforcement of SWCNTs with different diameters, whereas the $(8,8)$ SWCNTs reinforced EPON862 resin composites exhibited the highest enhancement of the Young's modulus. Chakraborty et al. [15] investigated the properties of the composites of pure monomer and trimer polycarbonate and their mixtures with different weight percentages of embedded SWCNTs at different temperatures. It was seen that the diffusivity of solvent molecules decreased with increasing percentage of CNTs at a specific temperature; and the polymerization played a role in enhancement of binding energy.

In this study, molecular dynamic (MD) simulations werecarried out to predict the thermo-mechanical properties of the curedepoxy network composed of diglycidyl ether bisphenol A(DGEBA) epoxy resin and tetrahydrophthalic anhydride (THPA) curing agent and single-walled carbon nanotubes (SWCNT) reinforced the epoxy matrix composites, respectively. Density of the materials and mean square displacements (MSDs) were calculatedto estimate the glass transition temperatures (Tgs) of the materials.

\section{Molecular Dynamic Simulation}

MD simulations were conducted using the Materials Studio 5.5 (Accelrys Inc.) software. COMPASS force-field was used in the simulation, which has been shown to provide accurate predictions of thermo-mechanical properties of thermosetting polymers $[3,4,10]$. The non-bond interactions with a cutoff distance of $9.5 \AA$, including van der Waals and electronic static forces, were applied. The atom approach was used for the dispersion interactions.

The cured epoxy network is composed of DGEBA resin and THPA curing agent. Chemical structures of the resin and hardener segments are shown in Figure 1. During the curing reaction, the acid anhydride groups of the curing agent molecules reacted with the epoxide groups of the epoxy resin. Initially, one THPA molecule reacted with one epoxide group. As the reaction contin- ued, more cross-links were generated between the epoxy resin and the curing agent. The cross-linking activity expanded in all directions and formed a network of macromolecules. Schematic of the curing reactions are shown in Figure 2.

In this study, the polymer model was dynamically built by assumptions. Firstly, 8 epoxy segments and 16 hardener segments containing reactive sites with a density equal to $1.2 \mathrm{~g} / \mathrm{cm}^{3}$ were packed into a 3D periodic cell box using the Amorphous Cell tool. This formulation was then mixed using the ensembles of the constant number of particles, constant volume and constant temperature (NVT) MD simulation for 100 ps performed at $298 \mathrm{~K}$ after initial molecular minimization (MM) based

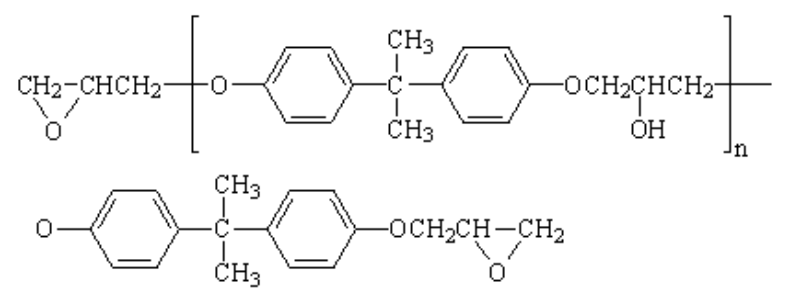

(a)

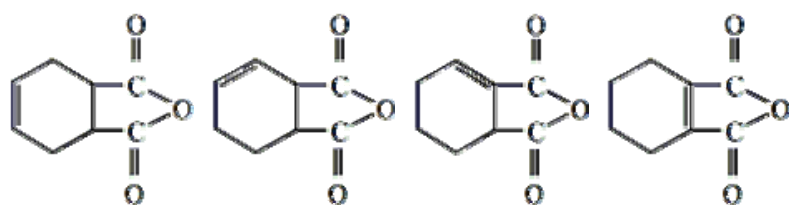

(b)

Figure 1. Chemical structures of the resin, hardener. (a) DGEBA; (b) THPA.

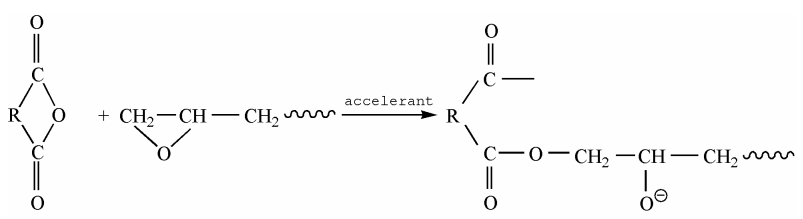

(a)

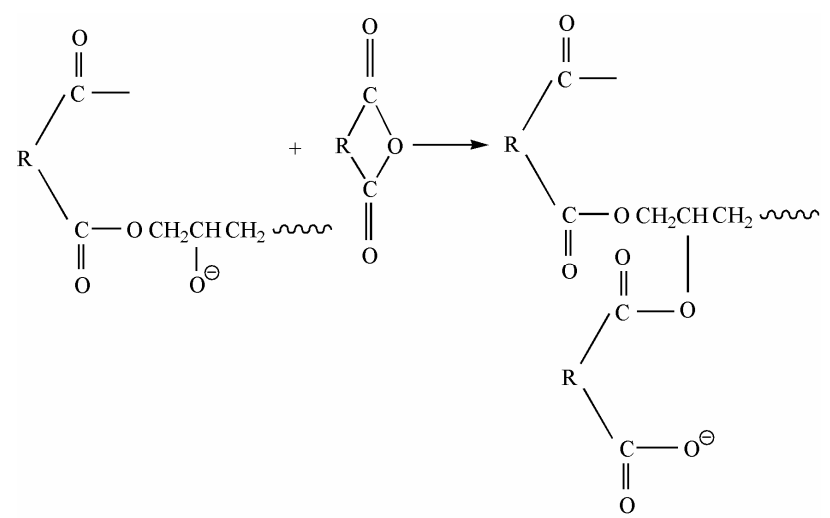

(b)

Figure 2. Schematic of the curing reactions. 
on COMPASS force-field. Under close proximity, covalent bonds were formed between the nearest reactive pairs within the reaction cutoff distance of $9.5 \AA$ considering the removal of ring catenation or spearing. Repeating these steps to form bonds for several times, a $19.33 \AA$ $\times 19.33 \AA$ × $19.33 \AA$ cross-linked polymer network with a conversion of $80 \%$ was finally obtained. For the CNT composites, a zigzag SWCNT $(4,0)$ with the diameter of $3.13 \AA$ and length of $34.08 \AA$ consisting of 196 atoms is embedded into the above amorphous DGEBA-THPA systems with a density equal to $1.0 \mathrm{~g} / \mathrm{cm}^{3}$, followed by the cross-linking activities. The resulting model system has a cell dimension of $21.52 \AA \times 21.52 \AA \times 21.52 \AA$ with a conversion of $60 \%$. Periodic boundary conditions are applied in all three directions for both models.

After the initial microstructure was generated, the minimization of the potential energy of the model was carried out, where COMPASS force-field was used. Then simulated annealing was performed by raising the temperature from $500 \mathrm{~K}$ to $900 \mathrm{~K}$ and cooling down to 500 $\mathrm{K}$ with steps of $20 \mathrm{~K}$ and an annealing time of 50 ps. After that, geometric optimization of 10000 steps and NVT MD simulation of 200ps were performed at a temperature above the Tg, such as $523 \mathrm{~K}$ in Forcite modules, and repeated for several times to relax the polymer chains.

In order to imitate the thermal performance in a kinetic process, simulation of cooling process was performed. The system was cooled stepwise from $523 \mathrm{~K}$ to $283 \mathrm{~K}$ with the rate of $10 \mathrm{~K} / 200 \mathrm{ps}$. At each temperature, 10000 steps geometric optimization simulation was performed to relax the polymer chains, followed by the ensembles of the constant number of particles, constant pressure and constant temperature (NPT) MD simulation under a pressure of $0.1 \mathrm{MPa}$ for $200 \mathrm{ps}$ to obtain the optimized density. In terms of non-bonding interaction treatments, atom-based direct cutoff of $9.5 \AA$ and a buffer of $0.5 \AA$ were used depending on the accuracy and efficiency of the computation. Velocity Verl et al. gorithm with a time step of $1 \mathrm{fs}$ was used for the integration of the atom motion equations throughout all simulations. Nose thermostat and Anderson barostat with cell time constant of 1.0 ps have been adopted. Each subsequent simulation was started from the final configuration obtained at the preceding temperature. The simulation in each case was performed with an interval of 1 femtosecond (fs) in each simulation step.

\section{Results and Discussion}

Various energies can be calculated from the MD simulations, which are used to analyze the roles of them in glass transition. The simulated results of total energy, potential energy and kinetic energy of the system against the tem- perature are plotted in Figure 3. It can be seen that the plots of these energy increases almost linearly with increasing temperature in the whole temperature range, indicating that the total energy, potential energy and kinetic energy play no distinctive roles in the glass transition process of the epoxy resin and its CNT composites.

Densities of the epoxy resin and the composites at room temperature were calculated to be $1.228 \mathrm{~g} / \mathrm{cm}^{3}$ and $1.012 \mathrm{~g} / \mathrm{cm}^{3}$, respectively, which are in good agreement with the appointed values. The evolution of density as a function of temperature of the DGEBA-THPA/SWCNT composites is plotted in Figure 4. A steady increase of the density with decreasing temperature and a clear change in the slope of the density curve were observed. The kink in the density vs temperature slope is defined as the

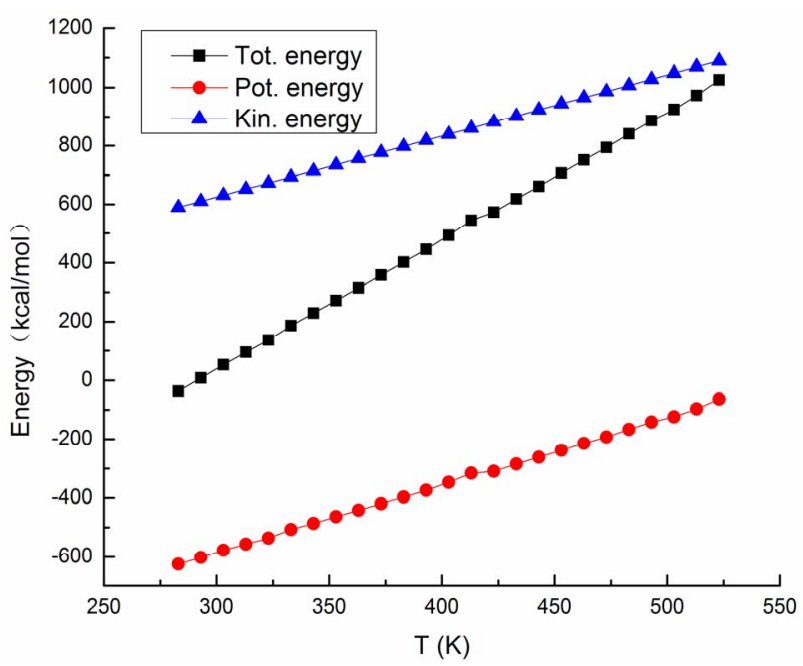

(a)

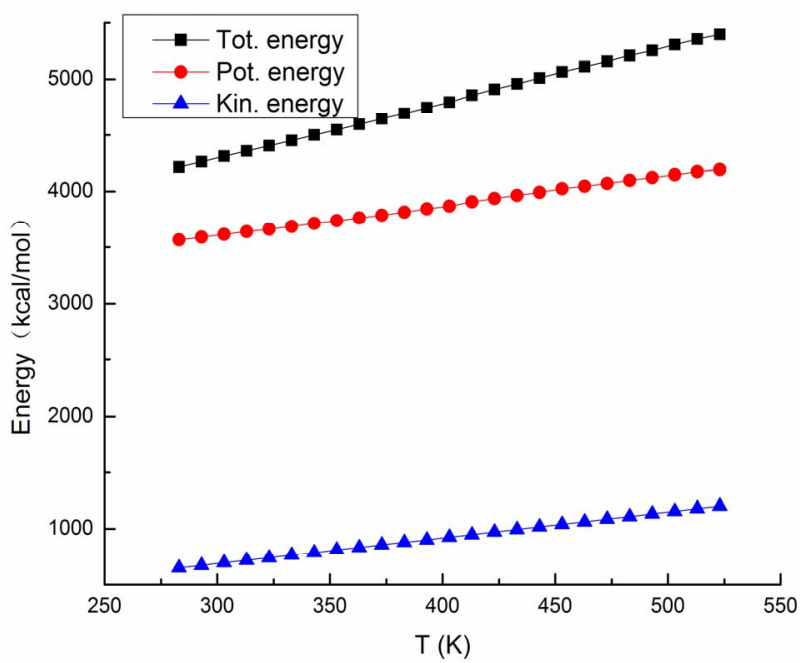

(b)

Figure 3. Energy variations as a function of tem- perature. (a) DGEBA-THPA polymer; (b) DGEBA-THPA/ SWCNTs composites. 
values of Tg, which occurs at approximately $423 \mathrm{~K}$, where the epoxy resin passes from the glassy state to the rubbery state.

The cross-reactions in the epoxy resin make significant differences in structure and properties from other linear polymers. It is instructive to analyze the motion of these cross-links in the model systems as a function of temperature. Because of incomplete reaction, segments on the polymer chains can be classified as cross-linked and free ones. Generally, the cross-linked segments exhibit much lower mobility than the free ones due to topological constraints. The value of Tg is correlated well with the polymer rigidity, namely the segmental mobility in polymer chain. The mean square displacements (MSDs) of the epoxy resin chains at 30 ps were calculated, which is plotted in Figure 5. The turning point of MSDs indicated Tg of the DGEBA-THPA polymer, which occurs around $365 \mathrm{~K}$.

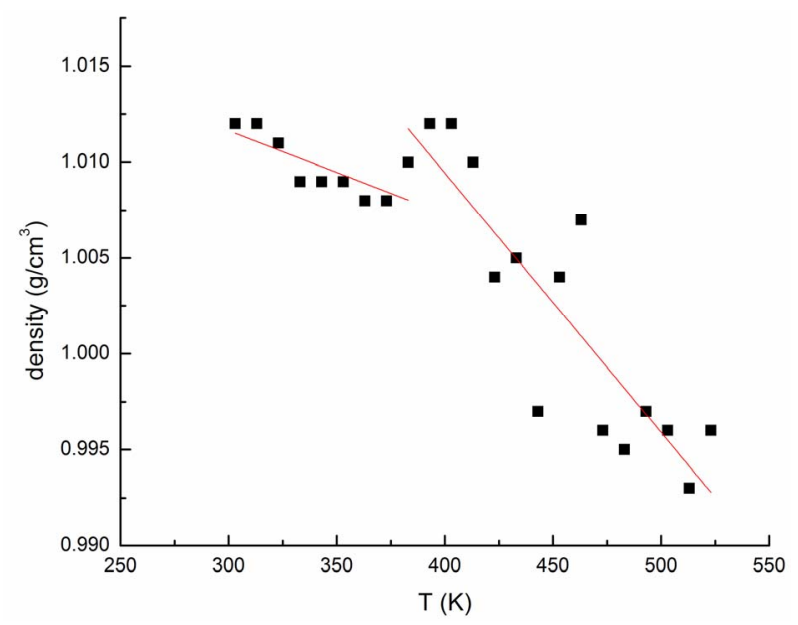

Figure 4. Density of DGEBA-THPA/SWCNT composites variation as a function of tem- perature.

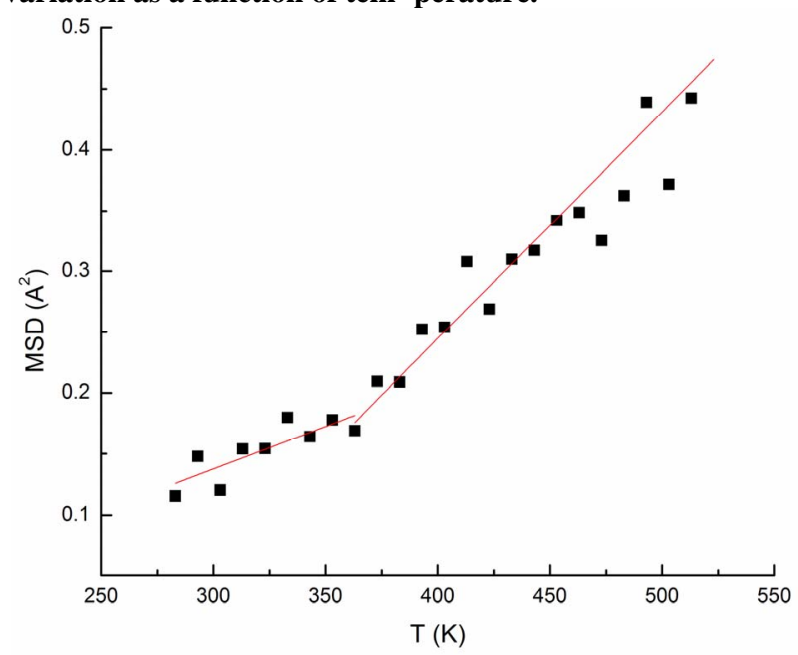

Figure 5. MSDs of the epoxy resin chainsvariation as a function of temperature.
From the MD simulations, Tg of the DGEBA-THPA/ SWCNT composites is much higher than the DGEBATHPA polymer, which is consistently with the fact.

It is very inspiring to see that the MD simulation method can be successfully applied to investigate the thermomechanical properties of the CNT/polymer composites. Once established, this approach can be applied more widely to a large group of candidate polymers and nanocomposites, proving a much more cost-effective way to narrow the fabrication and testing efforts to the selectedbest performers predicted by the MD simulations.

\section{Conclusion}

In the current study, two models of DGEBA-THPA cross-linked resin and its CNT composites were investigated by MD simulations. Different characters were calculated for the two models. The calculated results showed that the Tg of the two systems are $365 \mathrm{~K}$ and 423 $\mathrm{K}$, respectively, whereas the value of latter is much higher than the former, which is consistent with the fact. It can be expected that the method would be employed in further molecular simulation of structure and properties for cross-linked epoxy resin or other cured polymer network and their CNTs composites.

\section{Funding}

The present work is supported by the Key Projects of National High-tech R\&D Program of China (863 Program) under Grant No. 2012AA03A205, Hunan Province Technology Major Project of China under Grant No. 2011FJ1001.

\section{REFERENCES}

[1] B. F. Abu-Sharkh, "Glass Transition Temperature of Poly(vinylchloride) from Molecular Dynamics Simulation: Explicit Atom Model Versus Rigid $\mathrm{CH}_{2}$ and $\mathrm{CHCl}$ Groups Model," Computational and Theoretical Polymer Science, Vol. 354, No. 11, 2001, pp. 29-34. http://dx.doi.org/10.1016/S1089-3156(99)00070-7

[2] C. F. Wu and W. J. Xu, “Atomistic Molecular Modelling of Crosslinked Epoxy Resin,” Polymer, Vol. 47, 2006, pp. 6004-6009.

http://dx.doi.org/10.1016/j.polymer.2006.06.025

[3] C. F. Wu and W. J. Xu, "Atomistic Molecular Simulations of Structure and Dynamics of Crosslinked Epoxy Resin,” Polymer, Vol. 48, 2007, pp. 5802-5812. http://dx.doi.org/10.1016/j.polymer.2007.07.019

[4] H. B. Fan and Matthew M. F. Yuen, "Material Properties of the Cross-linked eEpoxy Resin Compound Predicted by Molecular Dynamics Simulation,” Polymer, Vol. 48, 2007, pp. 2174-2178.

http://dx.doi.org/10.1016/j.polymer.2007.02.007 
[5] C. Y. Li and A. Strachan, "Molecular Simulations of Crosslinking Process of Thermosetting Polymers," Polymer, Vol. 51, 2010, pp. 6058-6070. http://dx.doi.org/10.1016/j.polymer.2010.10.033

[6] C. Y. Li and A. Strachan, "Molecular Dynamics Predictions of Thermal and Mechanical Properties of Thermoset Polymer EPON862/DETDA,” Polymer, Vol. 52, 2011, pp. 2920-2928. http://dx.doi.org/10.1016/j.polymer.2011.04.041

[7] G. M. Odegard and A. Bandyopadhyay, "Molecular Modeling of Crosslink Distribution in Epoxy Polymers," Modelling and Simulation in Materials Science and Engineering, Vol. 20, 2012.

[8] A. Bandyopadhyay, "Molecular Modeling of EPON 862DETDA Polymer," Ph.D. Thesis, Michigan Technological University, Houghton, 2012.

[9] N. B. Shenogina, M. Tsige, S. S. Patnaik and S. M. Mukhopadhyay, "Molecular Modeling Approach to Prediction of Thermo-Mechanical Behavior of Thermoset Polymer Networks,” Macromolecules, Vol. 45, 2012, pp. 5307-5315. http://dx.doi.org/10.1021/ma3007587

[10] N. B. Shenogina, M. Tsige, S. S. Patnaik and S. M. Mukhopadhyay, "Molecular Modeling of Elastic Properties of Thermosetting Polymers Using a Dynamic Deformation Approach,” Polymer, Vol. 54, 2013, pp. 3370-3376. http://dx.doi.org/10.1016/j.polymer.2013.04.034

[11] S. Iijima, "Helical Microtubules of Graphtic Carbon," Nature, Vol. 354, No. 7, 1991, pp. 56-58. http://dx.doi.org/10.1038/354056a0

[12] J.H. Gou, B. Minaie, B. Wang, Z. Y. Liang and C. Zhang, "Computational and Experimental Study of Interfacial Bonding of Single-walled Nanotube Reinforced Composites,” Computational Materials Science, Vol. 31, 2004, pp. 225-236. http://dx.doi.org/10.1016/j.commatsci.2004.03.002

[13] Z. Y. Liang, J. J. Gou, C. Zhang, B. Wang and L. Kramer, "Investigation of Molecular Interactions Between $(10,10)$ Single-walled Nanotube and Epon 862 Resin/DETDA Curing Agent Molecules," Materials Science and Engineering A, Vol. 365, 2004, pp. 228-234. http://dx.doi.org/10.1016/j.msea.2003.09.032

[14] R. Mittal, M. Rastogi, N. Mahatele and A. Vidhyarthi, "Molecular Modeling for Calculation of Mechanical Properties of EPON862/Swcnts Composites,” IEEE, 2011.

[15] S. Chakraborty and S. Roy, "Structural, Dynamical, and Thermodynamical Properties of Carbon Nanotube Polycarbonate Composites: A Molecular Dynamics Study," Journal of Physical Chemistry B, Vol. 116, 2012, pp. 3083-3091. http://dx.doi.org/10.1021/jp212220m 